\title{
La question nationale, l'ethnicité et l'état en Afrique: Le cas du Cameroun
}

\author{
Par Ibrahim Mouiche
}

\section{Introduction}

Le départ de l'administration coloniale a posé avec beaucoup d'acuité le problème de l'intégration politique des pays africains qui, dès leur berceau, portaient les stigmates de "l'Etat segmentaire"; Etat où généralement, le gouvernement central coexiste avec des unités périphériques et centrifuges de pouvoir sur lesquelles il n'exerce qu'un contrôle relatif. Or, cette intégration politique à laquelle ils aspiraient, suppose à la suite de Myron Weiner cinq éléments:

- l'émergence d'une autorité centrale qui se place au-dessus des foyers périphériques,

- l'existence d'un sentiment national d'appartenance à un territoire qui supplante et élimine les solidarités paroissiales,

- l'établissement d'un lien entre gouvernants et gouvernés,

- l'apparition d'un sens minimum de consensus nécessaire au maintien de l'ordre social,

- et la promotion au sein de la population d'un esprit de concertation.

De là, M. Fogui ${ }^{1}$ distingue sur le plan analytique, la dimension horizontale de l'intégration politique (voir les deux premiers éléments) et sa dimension verticale (voir les trois derniers éléments): la première insiste sur la création d'une autorité centrale capable de s'imposer aux forces centrifuges, alors que la seconde vise à amortir le choc entre le centre et la périphérie et à promouvoir une culture qui ne sera plus paroissiale mais de participation. L'une jacobine et brutale semble condamnée à fonder les rapports de pouvoir sur la relation domination - soumission. L'autre plus subtile et plus profonde aspire à une société de négociation et de concertation voire un Etat de droit où les rapports de pouvoir seraient fondés sur la relation commandement - obéissance.

Les dirigeants africains vont plutôt mettre l'accent sur la formule jacobine, ce qui conduira au Cameroun à la quasi-diabolisation de la périphérie par les régimes du Président Ahmadou Ahidjo (1958-1982) et de son successeur M. Paul Biya (en poste depuis 1982). D'où la mise sous boisseau des identités ethniques. Seulement, la libéralisation politique

Fogui, J. P., L'intégration politique au Cameroun. Une analyse centre-périphérie, Paris, LGDJ, 1990 , p. $21-22$. 
des années 1990 a vu la violence s'installer et les sentiments ethniques voire "tribalistes" s'éparpiller au point d'atteindre même les milieux académiques et ecclésiastiques dans notre pays. ${ }^{2}$ Ce qui est une preuve que la paix sociale et la cohésion entre les quelques deux cent composantes ethniques n'étaient jusqu'ici maintenues que grâce à un système autoritaire de parti unique.

Face à ces données, un constat s'impose à savoir que le Cameroun a échoué dans sa tentative de construction de l'Etat-nation. En effet, l'unité nationale parce que de façade et artificiellement entretenue, n'a pas résisté aux pressions des forces centrifuges. Et l'on doit avoir l'honnêteté d'admettre qu'un changement de cap s'impose à défaut de tout recommencer. Il semble qu'il faille lier ces événements à la remise en question au plan mondial d'une problématique de construction nationale privilégiant les grands ensembles par rapport aux petites entités homogènes. Il semble qu'il faille poser avec sérénité mais dans toute son acuité, le problème de la balkanisation des nations africaines précoloniales telle qu'opérées par les différentes puissances coloniales et envisager leur intégration harmonieuse et leur coexistence pacifique.

Cette étude tourne autour de deux pôles: d'une part, il met en exergue la vacuité des politiques de construction nationale adoptées au lendemain des indépendances en Afrique (et plus particulièrement au Cameroun), et de l'autre, il propose une autre formule pour la gestion des sociétés plurielles africaines.

\section{De l'échec des politiques de construction nationale au Cameroun}

Si nous considérons la scène d'un œil froid, qu'avons-nous aujourd'hui en Afrique comme cadres administratifs de vie et comme réalités sociologiques? Des Etats sans nations, auxquels on a confié l'absurde tâche de mettre au monde ce qui aurait dû leur servir d'origine; des pseudo-nations constituées des ensembles ethniques juxtaposés que n'unit aucun lien organique; des peuples sans Etats; des dictatures ou des régimes mi-tribaux, mi de classe qui craignent en permanence sécessions, coups d'Etat ou révoltes populaires; des masses paysannes, éternelles vaches à lait d'élites urbaines, marginalisées qui croupissent dans ce que les économistes appellent poliment "l'économie informelle"; des bourgeoisies bureaucratiques sans aucun esprit d'entreprise qui considèrent l'Etat comme une caverne

Voir Ndi Mbarga, V., Ruptures et continuités au Cameroun, Paris, L'Harmattan, 1993, p. 113 et seq.; Collectif Changer le Cameroun, Le Cameroun éclaté? Anthologie des revendications ethniques, Yaoundé, Edition C3, 1992, et Ethnies et développement national. Actes du Colloque de Yaoundé, Yaoundé, Edition du CRAC, 1994. 
d'Ali Baba où se servir et puiser les ressources servent au patronage politique qui les maintiendra au pouvoir. ${ }^{3}$

En fait, le dilemme et la tragédie de l'Afrique et du Cameroun en particulier, résultent de la mise en place suite à la conquête coloniale du continent par des puissances extérieures au $\mathrm{XIX}^{\mathrm{e}}$ siècle, d'entités administratives réunissant différents groupes ethniques de manière arbitraire et à l'intérieur des frontières qu'ils n'avaient eux-mêmes fixées. En règle générale, ces nouvelles entités administratives n'ont tenu aucun compte des structures antérieures des sociétés qu'elles régissaient, allant parfois jusqu'à faire éclater certains groupes ethniques occupant des territoires voisins mais régis par des puissances distinctes. En d'autres termes, les nouvelles structures étatiques étaient totalement étrangères à l'Afrique sur le plan de leur conception et de leur organisation, et n'avaient aucune chance de constituer une base valable permettant de mobiliser la loyauté et l'énergie des populations concernées. ${ }^{4}$ C'est pourquoi il existe autant de nations dans les petits états comme le Gabon, le Togo, le Ghana qu'il y en a dans les grands états tels que la République démocratique du Congo, le Nigeria, le Soudan ou l'Angola bien qu'on les appelle de façon bizarre "tribus" ou "ethnies".

La résultante de ces politiques publiques et des stratégies par lesquelles les Africains y ont répondu n'est autre que l'ethnicité. Une pléthore d'ouvrages anthropologiques ou historiques ont démontré que les sociétés précoloniales étaient presque toujours pluriethniques et abritaient une grande diversité de répertoires culturels, que les principales formes de mobilisation sociale ou religieuse étaient transethniques. Cela ne veut pas dire que l'ethnicité soit une pure "construction" du colonisateur soucieux de diviser pour mieux régner, comme le soutiennent encore volontiers les nationalistes africains. En réalité, les colonisés ont participé à sa "formation" en s'appropriant les nouvelles ressources politiques, culturelles et économiques de l'Etat bureaucratique. L'importance politique de l'ethnicité provient alors justement de ce qu'elle est un phénomène éminemment moderne, lié à "l'Etat importé" , et non un résidu ou une résurgence de la culture traditionnelle. ${ }^{5}$

Prunier, G., Violence et histoire en Afrique, in: Politique africaine, n 42 , juin 1991, p. 11.

Balogun, O., La société tribale est-elle un atout ou un handicap pour 1'Afrique, in: Le Courrier ACP-CEE, Bimestriel n ${ }^{\circ}$ 140, juillet/août 1993, p. 61; Michalon, Th., Quel Etat pour l'Afrique, Paris, Présence africaine $\mathrm{n}^{\circ} 107,3^{\mathrm{e}}$ trimestre 1978, p. 18 et seq.; Tshiyembe Mwayila, L'Etat postcolonial, facteur d'insécurité en Afrique, Paris, Présence africaine, 1990, p. 15 et seq.

Bayart, J. F. , L'illusion identitaire, Paris, Fayard, 1996, p. 43 - 44. 
En Afrique, tous les systèmes de parti unique et tous les gouvernants durent donner des gages à une certaine démocratie formelle en organisant une représentation plus ou moins proportionnelle des régions, des ethnies ou des clans. Ils prétendirent ainsi justifier la représentativité politique des principales composantes des "forces vives de la nation", comme on disait et comme on dit encore aujourd'hui. Toutefois, la question des enjeux fut complètement évacuée au profit des tactiques plus ou moins réussies consistant à monter ces "composantes" les unes contre les autres pour la plus grande gloire du "Grand homme" et/ou de l'oligarchie dirigeante. Le factionnalisme ethnique, régional ou clanique a donc été et reste une réalité dure des régimes politiques africains qui n'ont jamais eu en fait la stabilité qu'on leur prêtait; l'équilibre fut constamment instable dans cette Afrique de la nuit qui a toujours su si bien se cacher pendant le jour. Tout le système de pouvoir reposa en définitive sur le mélange d'habileté, de terreur et de populisme que le prince parvint à mettre en œuvre pour contenir les rivalités dans les limites d'une paix civile acceptable mais précaire.

Avec plus de deux cents groupes ethniques, autant de langues et d'usages coutumiers, avec une constellation de religions dérivées du christianisme, de l'islam et de l'animisme, avec plus de cinq régions écologiques sous-tendues par des nuances géo-climatiques, démographiques et agro-pédologiques et ce malgré les velléités uniformisatrices de l'Etat moderne à travers les langues officielles importées et une centralisation abusive du pouvoir administratif et politique ${ }^{6}$, le Cameroun a toujours été et reste une mosaïque dont chaque élément rentre en interaction dans le schéma de la mobilité des populations depuis les temps lointains. En fait, le foisonnement ethnique apparaît ici comme un véritable obstacle à l'avènement de la nation; surtout que l'exacerbation des tensions inter-ethniques suscitées ou attisées, éloigne chaque jour l'horizon de celle-ci en même temps qu'elle accentue les réflexes identitaires.

Donc, si le Cameroun forme à l'évidence une grande société d'hommes et de femmes rassemblés sous les mêmes lois, il doit réactiver le vouloir-vivre en commun de ses communautés dont l'effritement fait apparaitre aujourd'hui l'horizon comme indépassable. D'ailleurs, en dépit du phénomène migratoire qui a favorisé les contacts entre les différentes composantes sociologiques du pays, il ne s'est opéré ni une intégration interethnique, ni l'émergence d'une unité linguistique au niveau national; et les courants migratoires spontanés ou imposés par des communautés guerrières puissantes et aguerries ont conduit plutôt à une juxtaposition d'entités ethniques les unes à côté des autres, repliées chacune sur son identité et entretenant peu ou presque pas de commerce culturel entre

6

Avec la réforme du 18 janvier 1996, le Cameroun est devenu un Etat unitaire décentralisé, mais ce train de réformes n'est pas encore appliqué. 
elles $^{7}$. Ainsi, les ethnies camerounaises se côtoient mais se méconnaissent; ainsi, le Cameroun est-il un "village" dont les habitants s'ignorent; d'où les préjugés tenaces qui engendrent méfiance et mépris; d'où les rivalités nourries aux complexes de toutes sortes qui se réactivent périodiquement et mettent les différentes composantes de la communauté nationale en situation de veillée d'armes.

Pour la commodité de l'analyse, nous allons ramener ce procès de construction nationale au Cameroun à deux thèses dont l'une extrémiste et l'autre médiane.

\section{La thèse extrémiste}

Cette thèse voit dans les politiques "d'unité nationale" du Président Ahidjo et "d'intégration nationale" du Président Biya, une clause de style si ce n'est un slogan creux. Elle est surtout le fait des auteurs bamiléké ${ }^{8}$, à l'instar de Jean-Louis Dongmo, de Victor Kamga, Emmanuel Kengne Pokam, ${ }^{9}$ du professeur Maurice Kamto, et plus récemment Kago Lélé etc.

Ainsi, dans un ton raciste et quasi-belliciste où il se livre à l'apologie de l'ethnie bamiléké, Emmanuel Kengne Pokam estime "qu'autant Ahmadou Ahidjo dans son discours prolixe prêchait inlassablement l'unité nationale, autant il ne ménageait aucun moyen dans sa pratique pour faire échec à cette unité". A preuve, "la primauté de l'intérêt particulier sur

Samuel Eboua, Ahidjo et la logique du pouvoir, Paris, L'Harmattan, 1993, p. 81, a raison pour dire qu'il faut un minimum de deux ou trois générations pour que le pays soit réellement bilingue; à la seule condition que la pratique du bilinguisme se généralise dans l'enseignement primaire, et que l'accent soit mis sur la pratique quotidienne de ces deux langues au lieu de se contenter de la traduction des thèmes et versions. Mais, en attendant, la partie anglophone du pays s'exprime en anglais, à peine en français; et la partie francophone en français, et difficilement en anglais. De part et d'autre, l'effort pour s'exprimer dans l'autre langue n'est perceptible que chez le personnel de l'administration et demeure pratiquement nul au niveau des masses. Même au niveau de l'administration, la loi du grand nombre jouant, l'impression qui prévaut est que les jeunes cadres anglophones font plus d'efforts pour s'exprimer en français que le font leurs collègues dans le sens inverse.

Sûrement pour défendre leur groupe ethnique dans la mesure où de par leur surface démographique et financière, de par leur capacité d'organisation, les Bamiléké croient fermement que d'autres groupes ethniques emploient des artifices pour leur barrer l'accès à l'Etat, parce que ces derniers redouteraient que du fait de cette hégémonie économique, ils n'assaillent définitivement leur hégémonie politique au Cameroun (thèse vraie jusqu'à un certain niveau); l'on ne doit pas également perdre de vue que la convivialité n'est souvent pas de mise dans les lieux d'immigration entre les autres ethnies et la diaspora commerçante bamiléké dispersée dans les principaux centres urbains du pays, ce qui souvent conduit à des violences intercommunautaires.

Kengne Pokam, E., La problématique de l'unité nationale au Cameroun, Paris, L'Harmattan, 1986. 
l'intérêt général" et surtout, "l'affirmation du fait ethnique au détriment du fait national" avec en prime "la mise en cause de l'entrepreneur camerounais", l'entrepreneur se ramenant à l'essentiel pour lui à l'homme bamiléké ${ }^{10}$.

Dans la préface à l'ouvrage de M. Emmanuel Kengne Pokam (1986), le Professeur Maurice Kamto va plus loin dans le ton:

"M. Ahmadou Ahidjo apparaissait en effet comme le chantre de l'unité nationale au Cameroun. Mais il s'agissait bien simplement d'une apparence car, à l'analyse, l'idée d'unité nationale a abouti à son contraire. Elle a été minée par l'absolutisme du pouvoir qui entraine l'intolérance politique, par l'artifice de "l'équilibre régional" et suprême fléau par l'ethnisme. Elle n'aura été qu'une arlésienne".

Dans Collectif Changer le Cameroun, ${ }^{11}$ la charge est encore plus lourde et la récrimination plus forte:

"La volonté de domination ethnique prenant alors le pas sur le désir d'intégration, le processus de la formation d'une nation au Cameroun piétine, d'autant plus que l'histoire ne cesse d'infliger de nouvelles blessures à ce peuple camerounais (...). En

L'on ne saurait expliquer le comportement économique des Bamiléké par le fait même qu'ils sont bamiléké car, être bamiléké ne signifie nullement posséder des qualités individuelles supérieures aux autres: vertu du travail, épargne, entraide, etc. Il s'agit là d'une tautologie qui n'explique rien. En fait, le comportement socio-économique des Bamiléké ne renvoie pas à une essence ethnique mais à un type de société où la plupart des statuts sociaux ne sont pas donnés par la naissance mais acquis par des initiatives individuelles dans un contexte d'anomie (au sens mentonnier du terme) généralisée; cf. I. Mouiche, Ethnicité et pouvoir au Nord-Cameroun, Verfassung und Recht in Übersee (VRÜ), 30 (1997), n 2, p. 182. De même, s'il est incontestable que les Bamiléké maitrisent les secteurs du commerce et du transport, il n'en demeure pas moins qu'ils ne contrôlent pas toute l'économie camerounaise comme le dit un autre chantre, en l'occurrence le Professeur Jean-Louis Dongmo (1981). D'ailleurs, tout le secteur tertiaire (qui inclut le commerce et le transport) ne représente qu'une part marginale de cette économie dominée plutôt par le secteur primaire. En effet, si le contrôle d'un secteur d'une économie (l'épicerie ici) suffisait pour dominer l'économie d'un pays, on en conclurait que ce sont les épiciers et les taxis arabes qui maîtrisent l'économie française, ou que ce sont les commerçants indo-pakistanais qui contrôlent l'économie britannique. En dehors des produits vivriers dont l'importance n'échappe à personne, mais qui ne sont pas quantifiés, l'économie repose également sur les produits de rentes (café, coton, cacao, etc). Par exemple l'élevage et la commercialisation de ses produits dérivés dont les ressortissants de la partie septentrionale du pays jouissent du monopole sont aussi importants. Il en va de même du coton. Ces mêmes " Nordistes" sont également présents dans le secteur du commerce et du transport. De même, le pays bamoun et les alentours de Foumbot, avec leurs sols volcaniques fertiles, sont réputés pour leurs productions vivrières, contribuant de façon significative à l'alimentation des centres urbains comme Douala ou Yaoundé. C'est le cas également du pays eton près de Yaoundé. D'ailleurs, la bourgeoisie d'affaires bamiléké se heurte dans la province anglophone du Sud-Ouest à la concurrence solide des Ibo (Nigérians) qui y sont les principaux détenteurs de la distribution.

Cf. Collectif Changer le Cameroun, 1994 (n. 2), p. 226 - 227. 
effet, aucune période de l'histoire de notre pays ne révèle une volonté patente de favoriser l'émergence d'un corps homogène habité par une âme qui anime ses habitants et les enracine dans toute partie de son territoire. Ahmadou Ahidjo faisait illusion: derrière le discours d'unité nationale, il a d'une part entretenu le clivage Nord-Sud au Cameroun, en limitant au strict nécessaire les contacts entre le Nord islamisé sous l'hégémonie peul et le Sud christianisé tenté par l'occidentalisation; il est révélateur à cet égard qu'après $1 / 4$ de siècle de règne sans partage, il n'ait pas décidé de relier ces deux parties du pays par des routes modernes (...). D'autre part, il a cherché une assimilation par soumission des populations non-peul et non islamisées du Nord à l'hégémonie foulbé afin de présenter le Nord d'apparence homogène face à un Sud dont il reconnaissait fort bien les divisions. En fait, il a davantage manoeuvré entre les ethnies qu'il ne s'est réellement attelé à l'édification de la nation camerounaise qu'il exaltait dans ses discours. Et le parti unique n'a nullement servi de creuset à l'unité nationale comme il le pensait, sauf en apparence. Il était en définitive le "père" d'une introuvable nation.

"Pareillement, le discours de "l'intégration nationale" qui a succédé à celui de "l'unité nationale" à partir de 1983 participe au Cameroun depuis 10 ans. De fait, le tribalisme n'a jamais été aussi vivace au Cameroun qu'aujourd'hui; théorisé puis érigé en doctrine de gouvernement, il a révélé la fragilité sociologique de l'Etat camerounais ainsi que l'éloignement de l'horizon de la nation chez nous."

A ces propos qui nous semblent excessifs, la thèse médiane est plus nuancée. Elle voit dans l'intégration politique au Cameroun, un processus d'interaction et de contradiction d'une part incarné par le pouvoir central, et d'autre part la société symbolisée par la périphérie. C'est un phénomène social englobant qui met en jeu les normes collectives, les modèles culturels, les dynamiques de variance et de la déviance. Dès lors, l'intégration politique peut être située à l'interface de l'Etat et de la société, elle est un processus à la fois intérieur et externe aux sociétés locales.

\section{La thèse médiane}

Elle est animée par Willard Johnson, Pierre-Flambeau Ngayap, ${ }^{12}$ Jean-François Bayart, ${ }^{13}$ Jean-Pierre Fogui, ${ }^{14}$ Michel Banock, ${ }^{15}$ Philippe Gaillard, ${ }^{16}$ Luc Sindjoun etc.

12

13

14

Ngayap, P.-F., Cameroun. Qui gouverne? De Ahidjo à Biya, l'héritage et l'enjeu, Paris, L'Harmattan, 1983.

Bayart, J. F. , L'Etat au Cameroun, Paris, Presses de la Fondation nationale des sciences politiques, 1985, $2^{\mathrm{e}}$ édition, et L'Etat en Afrique. La politique du ventre, Paris, Fayard, 1989.

Cf. Fogui (n.1). 
Suivant Pierre-Flambeau Ngayap, ${ }^{17}$ la recherche des grands équilibres géopolitiques a toujours été une constante dans la composition des gouvernements Ahidjo. Aussi veillait-il à ce que les représentations provinciales soient assurées, à ce que les grands équilibres géopolitiques soient respectés. Et, à l'intérieur de chaque province, il réalisait simultanément un micro-dosage qui se superposait aux macro-équilibres géopolitiques et permettait de mieux chevaucher les réalités socio-politiques que sont les ethnies. C'est ce que Jean-François Bayart a pu appeler à juste titre, le "processus d'assimilation réciproque" opéré de février 1958 à novembre 1982 par le régime Ahidjo; ces décennies ayant vu l'émergence d'une vaste alliance regroupant les différents segments régionaux, politiques, économiques et culturels de l'élite sociale.

Dans le même sens, les propos de Philippe Gaillard ${ }^{18}$ sont encore plus élogieux pour le régime Ahidjo:

"Socialisme africain, libéralisme planifié, développement autocentré, démocratie gouvernante. De congrès du parti " unifié" , l'intitulé de la doctrine changea. Peu importe: la phraséologie fluctuante n'était que l'habillage de circonstance d'un pragmatisme essentiel charpenté par le sens de l'Etat. Le succédané d'idéologie d'Ahmadou Ahidjo, son grand dessein tenait en deux mots; l'unité nationale (... ). La méthode était choisie: l'unification par le haut, par les structures. Toute l'histoire du Cameroun sous Ahidjo a été celle de l'unification de l'Etat et du parti, selon un processus dont les étapes ont été déterminées par le sens de l'opportunisme de celui qui en décidait (...).

Mais seulement, les mentalités ne pouvaient pas suivre à ce rythme. Les séquelles de la rébellion, les différences de cultures entre le Nord soudano-sahélien et le Sud bantou, comme entre les Anglophones et les Francophones, les contentieux et rivalités entre les quelques deux cents ethnies rendaient plus ardue que nulle part dans le monde, la fusion nationale. Les progrès furent pourtant spectaculaires et chacun s'identifia bientôt comme Camerounais-ajoutant certes la référence ethnique. La politique d'équilibre régional y contribua".

Jean-Pierre Fogui ${ }^{19}$ a donc raison pour dire que l'oeuvre d'intégration nationale accomplie depuis l'indépendance au Cameroun est un phénomène surtout institutionnel qui tarde à

Banock, M., Le processus de démocratisation en Afrique: le cas du Cameroun, Paris, L'Harmattan, 1992. Afrique livres, 1994. 
irriguer la société dans l'ensemble et à pénétrer les mentalités; car, l'on ne peut parler d'une intégration nationale que dans la mesure où le sentiment d'identification et d'attachement des populations se tourne vers un repère national et non exclusivement ethnique ou régional. D'où il convient de distinguer avec Willard Johnson entre la construction de l'Etat (State-building) et la construction de la nation (Nation-building).

Pour cet auteur (il est vrai, cette littérature date), le Cameroun a marqué des pas qualitatifs dans la construction de l'Etat mais pas la nation qui est plutôt une " construction sociale et historique problématique" suivant les termes de Michael Schudson. ${ }^{20}$

\section{L'Etat-nation, construction sociale et historique problématique}

"Le bien le plus précieux que nous transmettons les uns aux autres, c'est l'appartenance à une communauté humaine". La phrase est de Michael Walzer. Pour Edward Shils, "appartenir à une société politique est une nécessité humaine". Mais de quelle communauté s'agit-il? De quelle société politique? Aujourd'hui , alors que nous entendons parler de plus en plus d'institutions transnationales, de cultures frontalières, d'Etats qui se désagrègent, de sous-cultures et sous-nationalismes, de multiculturalisme et de métissage du monde, la question nous apparait dans toute sa complexité. Identifier les limites d'une société est une tâche si difficile qu'elle devrait nous amener à nous interroger sur l'habitude qu'ont les spécialistes des sciences sociales de prendre l'Etat-nation comme unité d'analyse, c'est de ne pas avoir vu " une construction sociale et historique problématique" ${ }^{21}$ aussi bien en Afrique qu'en Europe.

\section{Contre le modèle unitariste européen, le modèle africain est plural}

Pendant longtemps, en Occident comme en Afrique, on a cru que les institutions occidentales, en l'occurrence, l'Etat-nation sont transférables d'une société à l'autre, indépendamment de son histoire, de sa culture, de ses valeurs ou de ses institutions. Les résultats catastrophiques de cette croyance ne sont plus à démontrer mais il faut les expliquer. Si l'Etat de type occidental, transféré dans les autres aires socio-culturelles suscite des mouvements non pas d'intégration, mais de désintégration, c'est qu'il y a divergence, sinon incompatibilité radicale des valeurs et des significations sociales d'une sciences sociales, ${ }^{\circ} 133$, Paris, UNESCO/ères, août 1992, p. 79. 
société à l'autre et que la perception et la pratique de pouvoir ne sont pas les mêmes en Occident et en Afrique. ${ }^{22}$

L'Etat-nation a vu le jour dans la haute antiquité des peuples du monde d'Orient et d'Occident, à l'intérieur d'un contexte et d'une histoire qui avaient essaimé ses modèles dans ces champs culturels. L'Etat babylonien, persan ou gréco-romain, l'Etat judéochrétien et islamo-arabe ont un air de famille dans leur conception territorialiste et ethniciste de la construction politique. ${ }^{23}$ De ce fait, l'Etat-nation fut conçu en dehors de toute considération ethnique ou religieuse, son idéologie ambiante étant réductrice, unificatrice et universelle.

Importé en Afrique, il devait se trouver rapidement confronté aux difficultés d'administrer une population hétérogène par la langue, les comportements religieux ou les mœurs. Et, à des rares exceptions près, sa réponse fut partout la même: exalter l'homogénéité car qui dit Etat-nation, dit Etat unitaire. Un Etat, une souveraineté, une nation, un peuple, une patrie, une langue, une culture, une histoire. L'Etat-nation n'admet pas d'altérité dans ses manifestations extérieures, dans l'espace public. ${ }^{24}$ Dès lors, son introduction dans notre continent a constitué avec la colonisation une rupture totale dans la mesure où elle a été accompagnée de "logique et de mythologie" radicalement étrangères aux expériences africaines. Au modèle "logique" africain que nous dénommons "plural" et que nous retrouvons dans d'autres sociétés communautaristes, la modernité européenne a substitué un modèle nouveau. A l'image de Dieu catholique du Concile de Trente, omnipotent et omniprésent, mais extérieur et supérieur à ses créatures, on a pensé l'Etat, puis le marché, le code, le territoire ou la personne. Ce modèle est dit " unitariste" parce qu'il repose sur la réduction des instances sociales dans l'unité imposée de l'institution qui les incarne. ${ }^{25}$.

En d'autres termes, l'idéologie de l'Etat-nation indo-européen et judéo-chrétien fait de l'Etat l'essence territoriale d'une ethnie usant des rapports de force qu'il régente à sa philosophie et à sa vision du monde, par une politique de négation de l'autre, l'assimilation; mais dans le contexte socio-politique de l'Afrique noire, aucune ethnienation ne peut réaliser cette prouesse et cela explique pourquoi à tort ou à raison, toute tentative du pouvoir africain de s'identifier à une ethnie-nation provoque immédiatement des sursauts de révolte réelle ou diffuse de la part d'autres ethnies. D'ailleurs, l'on peut se

Mappa, S., Pouvoirs traditionnels et pouvoir d'Etat. L'illusion universaliste, Paris, Karthala, 1998, p. 10. Tshiyembe Mwayila (n. 4), p. 90.

24 Guyonmarc, C., Les facteurs à la base des préjugés, in : Le Courrier ACP-CEE, Bimestriel, $\mathrm{n}^{\mathrm{O}}$ 140, juillet/août 1993, p. $52-53$.

25

Birnbaum, P., Nationalismes: la comparaison France / Allemagne, in: Revue internationale des sciences sociales, $n^{\circ}$ 133, Paris, UNESCO/ères, août 1992, p. 423 - 432; Michalon (n. 4). 
demander légitimement si le processus de création de la nation, c'est-à-dire une expression ethnique bien stabilisée et cristallisée dans sa durée peut être une orientation politique d'un Etat ou d'un parti. La question est particulièrement évidente pour l'Afrique noire. Nombre de fronts nationalistes n'ont-ils pas proclamé que c'est dans les conditions de la lutte imposant l'unité contre l'ennemi colonial que se sont forgés les sentiments d'unité nationale? Qu'unifiant les groupes ethniques, la lutte de libération avait ainsi créé la nation?

Cette vision ne correspond pas à la réalité. Il convient tout d'abord de revenir sur le sens du mot" nationalisme" dont on peut dire simplement qu'il est l'expression politique possible d'un sentiment national. Ainsi, la nation grecque, la nation serbe, la nation polonaise, la nation chinoise existaient lors des luttes contre l'occupant étranger. La lutte de libération nationale visait donc à rendre possible la pleine expression de la nation par sa construction en Etat. Il s'agit d'un nationalisme produit par une nation préexistante. Or, la situation est tout à fait différente dans de nombreux pays noirs africains. L'on a qualifié les luttes d'émancipation anticoloniale, de luttes de libération nationale par analogie légitimante envers le processus européen du siècle précédent. Or, si des consciences ethniques diverses existaient, si le bien-fondé social de cette lutte ne saurait être mis en cause (exploitation et violence coloniales), il n'y avait le plus souvent nulle nation. Les fronts de libération ont proclamé que la lutte avait unifié le peuple... Dit ainsi, c'est une contrevérité. Il n'y a qu'à voir les exemples bissau-guinéen, angolais, mozambicain , algérien et même camerounais pour des cas de lutte armée. Toute guerre provoque des processus unificateurs et simultanément diviseurs. Par ailleurs, ce n'est point en quinze ans de lutte, fut-elle armée que se crée une nation. Les fronts étaient nationalistes non pas dans la mesure où ils avaient exprimé une nation existante, mais dans celle où ils combattaient non seulement pour la création d'un Etat indépendant, mais pour la création d'une nation. La nature de ce nationalisme est totalement différente: il n'est point produit par une nation, il n'est point massif, il est induit comme phénomène élitaire dans la lutte sociale anticoloniale de masse par une élite moderne universaliste. Ce nationalisme élitaire est antidémocratique dans la mesure où il veut imposer aux peuples présents au sein des frontières coloniales des caractéristiques "nationales" qu'ils ne ressentent pas. ${ }^{26}$.

Dans le cas du Cameroun qui nous intéresse principalement, comment pouvait-on rêver d'une République une et indivisible quand on sait que de par sa diversité ethnique, ce pays est un véritable "habit d'Arlequin", une Afrique en réduction, avec ses deux cent trente ethnies, avec ses peuplades négritiques, arabes, bantous et soudano-sahéliens? Doit-on également continuer à célébrer les louanges de l'Etat-nation quand en Europe, on chante 
son oraison funèbre? Ce continent ne s'oriente-t-il pas définitivement vers la décentralisation? 


\section{La crise de l'Etat-nation en Europe}

Comme les Européens subvertissent inconsciemment l'Etat dans le fait même de le proclamer souhaitable, les comparatistes de la sociologie historique sont en train de le rejeter involontairement à la périphérie tout en affirmant sa position centrale. Les appels à l'indépendance nationale venus ces dernières années de Georgie, d'Estonie, de Croatie, de Bosnie, etc., pourraient nous conforter dans l'illusion d'accéder enfin à l'âge où chaque peuple aura un Etat à part (en propre), l'apothéose de l'Etat-nation, la fin de l'histoire. Or, bon nombre d'indicateurs précurseurs laissent penser au contraire que nous allons au-delà de l'effervescence actuelle des nationalismes, vers un effondrement général de l'Etat fort, unifié, centralisé, aux frontières bien délimitées qui avait commencé à s'imposer en Europe au XVIII ${ }^{\mathrm{e}}$ siècle pour finir par servir de modèle au monde entier. La fluidité croissante du capital, de la main-d'œuvre, des marchandises, de l'argent et des pratiques culturelles fait qu'aucun Etat n'a plus d'emprise sur ce qui se passe à l'intérieur de ses frontières. ${ }^{27}$

A des rares exceptions près, comme la Norvège ou l'Irlande, l'idée d'un Etat-nation culturellement homogène a toujours été un mythe qui aura permis à des Etats hétérogènes comme la France ou l'Espagne de privilégier un segment et une perspective seulement dans les affaires nationales. Bientôt, le temps où tout Etat européen pouvait régler la circulation du capital, de la main-d'œuvre, des marchandises, de l'argent, des idées ou des pratiques culturelles à travers ses frontières, et où n'importe quel Etat pouvait garantir son homogénéité culturelle ne sera plus qu'un souvenir. Les idées, les prétentions et les pratiques des soi-disant Etats-nations disparaîtront d'elles-mêmes. Ces nations étendues au sens des populations liées par une culture, pourront fort bien survivre, prospérer et même se reformer, mais sans plus être rattachées à des Etats puissants ${ }^{28}$

Il est même permis d'ailleurs de douter que la culture soit un facteur d'intégration essentiel. $\mathrm{Du}$ reste, la plupart des sociologues et des historiens n'en font nullement le principal mécanisme d'intégration des sociétés nationales. Etudiant la Chine moderne, un chercheur (Shue) fait par exemple observer que, depuis 1949, le Parti communiste exerce son emprise sur la culture populaire des villages, mais que l'uniformité culturelle aura finalement le plus progressé, " non pas sous l'effet du matraquage idéologique qui a marqué les dernières années de Mao, mais pendant les années 50, lorsque, ont commencé la réforme agraire et l'effort de collectivisation". Edward Shils constate lui aussi que c'est à l'intégration économique et politique plutôt qu'aux facteurs culturels que l'on doit l'intégration des masses dans les Etats modernes. Même en Afrique, les crises paroxystiques du Rwanda, du Burundi et de la Somalie suggèrent que la continuité territoriale et culturelle entre les

Tilly, Ch., Prisonniers de l'Etat, in: Revue internationale des sciences sociales, $\mathrm{n}^{\circ} 133$, Paris, UNESCO/ères, août 1992, p. 373; Yameogo, H. (n. 26), p. 23.

28

Tilly, Ch. (n. 27), p. 374. 
sociétés précoloniales et les Etats contemporains ne constitue nullement un gage de stabilité. On peut même se demander, à partir de l'exemple des conflits de la région des grands lacs, si la modernisation idéologique et militaire des Etats d'origine précoloniale n'a pas favorisé la radicalisation des rapports sociaux en leur sein, là où leur incorporation dans des espaces politiques élargis, à la faveur du changement d'échelle généralement provoqué par la colonisation, a rendu inévitables des compromis entre les élites, voire leur assimilation réciproque, et a tendu à diluer les contradictions sociales, ou en tout cas à les amortir. Jean-François Bayart ${ }^{29}$, peut alors célébrer "l'illusion identitaire", considérer "le culturalisme comme idéologie de la globalisation" et se demander si la "culture serait un mot à jeter". Donc, l'important est de prendre en compte la problématique locale légitime en Afrique et au Cameroun en vue d'un Etat multicommunautaire.

\section{Mutations socio-politiques, rivalités ethniques et nécessité d'un état multicommunautaire au Cameroun ${ }^{30}$}

L'interrogation politique et scientifique sur l'identité est de retour. La profusion des titres ces dernières années en sont une illustration évidente. Outre les problématiques liées à des situations nationales, ce retour de l'identité à travers ses multiples avatars ethniques, régionaux, nationaux, communautaires et religieux, traduit à la fois la remise en cause des modèles institutionnels d'intégration et d'organisation unitaires à la française ou communautaristes à la nord-américaine, l'affaiblissement du modèle étatique occidental jusqu'ici largement considéré comme ayant vocation à l'universel et la déliquescence avancée des modèles développementalistes classiques postulant que la modernisation implique systématiquement la disparition de la tradition et des concepts identitaires "rétrogrades" qui lui étaient associés (ethnicité, culture paroissiale, communautarisme). ${ }^{31}$ Mais à côté de ce courant de pensée, existe une autre hypothèse différente. La montée des intégrismes et le retour des discours à caractères ethniques ou tribaux seraient la conséquence de l'adaptation (forcée) des économies des Etats-nations aux conditions d'une production internationalisée. L'argument précédent se trouve inversé. La réaffirmation des phénomènes identitaires devient l'indicateur d'un mal développement, c'est-à-dire d'un développement économique qui entraîne la marginalisation des franges plus importantes de la population. Les politiques économiques qui visent à faciliter la globalisation (les

30 Voir la conclusion de mon article sur "Mutations socio-politiques et replis identitaires en Afrique: le cas du Cameroun" in: African Journal of Political Science. Revue africaine de science politique, vol 1, n² 2, dec, 1996.

31

Darbon, D., Ethnicité et nation en Afrique du Sud. Imageries identitaires et enjeux sociaux, Paris, Karthala, 1995, p. 8. 
programmes d'ajustements structurels par exemple), en adaptant les Etats aux exigences d'une production internationalisée produisent en même temps les facteurs d'instabilité. ${ }^{32}$

Cette interrogation est d'autant plus urgente quand l'on sait que l'ethnicité est aujourd'hui au cœur des crises plus sanglantes non seulement en Afrique mais aussi dans l'exYougoslavie, les anciennes Républiques de l'ex-URSS (Arménie, Azerbaïdjan, Russie, Tchétchénie) et les régions indiennes de certains pays d'Amérique latine. L'ampleur des conflits liés à l'ethnicité (les massacres cruels au Rwanda, les violences quotidiennes au Burundi, au Congo démocratique et au Congo-Brazzaville) est telle que la question ethnique qui était perçue comme exclusivement africaine et relevant d'un répertoire prémoderne commence à être lue comme une des manifestations les plus spectaculaires du politique sur la scène internationale.

Néanmoins, cette crise de l'universalité de l'ordre politique ne va pas jusqu'à entamer la continuité de l'Etat en Afrique. En effet, l'adhésion des Africains aux cadres territoriaux légués par le colonisateur est l'un des traits saillants de l'histoire récente de ce continent. En dehors de l'Erythrée et dans une certaine mesure, le Somaliland, les exemples de sécession sont inexistants depuis le début du processus de démocratisation des années 1990. Les velléités sécessionnistes observées à Zanzibar, en Tanzanie, dans les provinces camerounaises anglophones du nord-ouest et du sud-ouest ${ }^{33}$, dans les provinces orientales du Congo-Kinshasa, etc., ne constituent pas des tendances lourdes. En lieu et place de la sécession, ce qui est envisageable à court terme, c'est "le droit à l'autodétermination interne". ${ }^{34}$ Même les guerres civiles ne remettent pas en cause l'unité nationale; même la voie fédérale a parfois été écartée avec violence par les protagonistes de ces conflits, partisans farouches d'un Etat unitaire, comme au Tchad, en Angola ou au Soudan. La seule véritable tentative de sécession a été celle du Biafra en 1967 et elle a été écrasée. ${ }^{35}$

C'est à ce niveau que l'ethnicité se démarque du nationalisme. En effet, le discours du nationalisme s'articule presque toujours autour des trois principes de base ainsi que le dit si bien Elie Kedounie. Tout d'abord, le premier affirme que l'humanité est naturellement divisée en nations. Ensuite, les caractéristiques distinctives de ces nations peuvent être établies. Enfin, le gouvernement des nations par elles-mêmes est le seul type de gouvernement légitime. Aussi le nationalisme est-il synonyme de revendication à l'autodétermination, à la création d'une communauté politique distincte et souveraine pour

32

33

34

35

Cf. n. 26.

Pour Piet Konings, Le problème anglophone, in: Politique africaine $\mathrm{n}^{\circ}$ 62, 1996, p. 25-34, demeure le problème crucial auquel fait face l'Etat camerounais postcolonial soucieux de forger un Etat-nation stabilisé.

Oeter, St., Selbstbestimungsrecht im Wandel, ZaöRV 52 (1992), p. 741/743 .

Cf. Bayart (n. 5), p. $45-46$. 
chaque nation, pour chaque peuple. Comme l'écrit Ernest Gellner "l'objectif du nationalisme est de faire coïncider les frontières de la communauté politique, c'est-à-dire à notre époque, l'Etat, avec les frontières de la nation". Au contraire, l'ethnicité n'entraîne pas cette quête de souveraineté politique par les groupes sociaux qui se définissent par une même identité ethnique. Ces groupes se contentent de revendiquer une reconnaissance spécifique à l'intérieur des frontières de l'Etat dans lequel ils vivent ou une reconnaissance qui dépasse les frontières de plusieurs Etats. ${ }^{36}$

\section{Libéralisation politique et rivalités ethniques au Cameroun}

Affaibli aujourd'hui par l'efficacité des mobilisations identitaires, l'Etat africain souffre consécutivement d'un grave déficit de citoyenneté imputable autant à sa légitimité précaire, à son extranéité qu'à sa faible capacité politique. L'ensemble de ces données tend à aggraver l'importance des " espaces sociaux vides" , c'est-à-dire des secteurs de la société que la scène politique officielle ne parvient ni à mobiliser ni à contrôler et au sein desquels se déploient des formes d'autorité de substitution qui captent à leur profit des allégeances individuelles. La prolifération de ces espaces tend aussi à faire reculer les frontières internes de l'Etat, à démultiplier et à entrecroiser les réseaux d'allégeance qui intégrant les individus. 37

Ainsi, entre 1991 et 1994, l'on a assisté au Cameroun à une véritable explosion interethnique qui s'est manifestée de plusieurs manières. Et d'abord par une sorte de débridement du discours ethnocentré avec des invectives à caractère "tribaliste" dans les lieux publics (marchés, services publics, bars, écoles, églises, etc.) :

"Toujours ces Bamiléké (...); vous les Bassa! Encore des Béti! Les Anglo alors! Quant aux Nordistes!"

Tout un discours agrémenté d'expressions les plus injurieuses qui faisait que le locuteur se comporte toujours en agresseur. Des réactions spontanées et instinctives, on était passé à des formes plus élaborées et plus motivées des revendications ethnocentrées. Des mémorandums, des essais politiques et philosophiques, des articles des journaux avaient circulé pour dénoncer ou redouter toute forme d'hégémonie (politique, économique, religieuse) de l'un ou l'autre groupe ethnique. Des groupes étaient montés au créneau pour sonner l'alarme de la marginalisation et de la persécution. Des chefs traditionnels s'étaient élevés pour clamer des revendications éminemment ethniques et récuser la portion congrue édition. 
du " gâteau national". ${ }^{38}$ Peu avant même l'autorisation des partis politiques par les lois de décembre 1990, les rivalités ethniques s'étaient même accentuées avec des accusations par certains journaux privés d'obédience bamiléké, notamment "Le Messager", "La Nouvelle Expression", "Challenge Hebdo", sur les détournements des fonds publics et les nominations des hauts responsables de l'Etat, accusation visant surtout les Béti, l'ethnie apparentée à celle du Président Biya.

Ce qui n'est pas étonnant en Afrique où le bilan de carrière d'un haut fonctionnaire se ramène souvent à la question de savoir quels avantages il a accordés aux siens. Les autorités étatiques le savent d'ailleurs, elles qui s'efforcent d'en tenir compte lors des nominations à des postes de responsabilités par l'institutionnalisation du principe hautement contestable de "l'équilibre régional". ${ }^{39}$ Un ministre qui quitte le gouvernement est généralement remplacé par un ressortissant de la même ethnie ou de la même région. En outre, le jeu est sans règles écrites et parfois, le chef de l'Etat privilégie le groupe ethnique auquel il appartient. Même en matière électorale, l'appartenance à la région, à l'ethnie, ou à la ville, constitue généralement le critère au nom duquel le candidat est jugé apte à défendre les intérêts de sa localité, de sa région, de son ethnie d'origine. Et lorsqu'apparaît une compétition entre deux candidats qui répondent à ce critère, on reprochera souvent à l'un d'eux d'avoir par une alliance antérieure impie avec des gens d'autres régions traditionnellement rivales trahi les intérêts sacrés de l'ethnie. ${ }^{40}$ C'est pourquoi l'on parle de plus en plus de la politisation de l'ethnicité ou de l'ethnicisation de la politique, l'objectif recherché étant l'accumulation.

En fait, la référence à des catégories d'identités primordiales demeure incontournable même si elle est devenue plus volatile dans les grandes métropoles. Etre de tel ou tel village, de telle ou telle région ou de telle ou telle ethnie rend aléatoire toute prise en compte d'enjeux autres que ceux relatifs au pouvoir. Cette genèse des identités particulières dans le nouvel espace politique n'est pas le rejet ou la négation de l'Etat, mais l'adaptation inventive aux changements radicaux qu'il représente, un mode d'appropriation de ses institutions et de partage de ses ressources. Cela est maintenant bien connu des africanistes, et R.H. Bates peut affirmer que, "les groupes ethniques sont une forme de coalition gagnante à la marge suffisamment large pour garantir des profits dans la lutte pour le

40 Collectif Changer le Cameroun, 1992, (n. 11).

Le concept d' "équilibre régional" présente beaucoup d'impertinences. Il suppose que les régions en tant qu'unités sont bien déterminées et que leurs frontières sont flexibles du fait qu'elles sont le produit des multiples négociations. Au contraire, le Cameroun est traversé par des clivages et, à l'intérieur de chaque région existent des tensions ethniques.

Hazoume, G.-L., Idéologies tribalistes et nation en Afrique: le cas dahoméen, Paris, L'Harmattan, 1972, p. $21-22$. 
partage des dépouilles, mais aussi suffisamment restrictive pour maximiser le rendement de ces profits".

Les documents écrits dans la vague de la grande explosion de 1991 ont porté à l'attention du grand public camerounais plusieurs aspects de problèmes économiques rencontrés par les porte-paroles informels ou autorisés des différentes composantes de notre peuple:

- d'abord les problèmes fonciers. Sous couvert de ceux-ci certains groupes ethniques, notamment les Bamiléké sont traités "d'envahisseurs"; d'autres sont décrits comme des paresseux incapables de mettre en valeur les terres qu'ils revendiquent en qualités d'autochtones;

- ensuite la répartition du patrimoine national. Beaucoup se plaignent d'être exclus de la gestion des richesses nationales. Les arguments principaux sont toujours appuyés par la sous-représentation dans l'appareil de l'Etat, la dénonciation de la politique de l'aménagement du territoire.

La Déclaration de Buéa ${ }^{41}$ sur les "problèmes anglophones" en est un exemple édifiant:

"Notre problème (...) vient du fait que les dirigeants francophones ont trahi notre confiance et qu'il n'y a pas de transparence dans les affaires publiques. Au cours de ces 32 années, notre accord a été violé. On nous a privés des droits électoraux, marginalisés, traités avec suspicion, on a cantonné à des fonctions non essentielles notre participation à la vie de la nation (...). A la suite de manœuvres et manipulations, nous sommes passés au statut de partenaires égaux à celui du peuple sous le joug".

Cette opposition "Anglophones-Francophones" ne doit pas occulter les rivalités bétibamiléké, celles opposant au Nord-Cameroun les Kirdi aux Peul voire les Arabes choa aux Kotoko dans la pointe septentrionale; comme la "révolte sawa" à Douala aux lendemains des municipales de 1996 dont les résultats affichaient de façon notable, l'hégémonie bamiléké sous la bannière du parti du SDF (Social Democratic Front), hégémonie que devait dénoncer avec véhémence le journal "Elimbi" d'obédience sawa.

Au sein même de la communauté anglophone, les conflits sont légion. Par exemple, M. Mope Simo a pu observer dans la plaine de Ndop (province du Nord-Ouest) les différentes formes de violences suscitées par des litiges fonciers entre plusieurs chefferies notamment Bafanji contre Bamunkumbit, Bamunkumbit contre Bali-Gashu, Bamunka contre ses voisins et Bali-Kumbat contre Bafanji. L'on peut également mentionner les antagonismes entre les provinces anglophones du fait de la peur qu'éprouve la minorité du Sud-Ouest de subir la domination du Nord-Ouest, du renforcement de la distinction du Sud-Ouest en raison de la richesse énergétique de son sous-sol et de la concurrence à laquelle se livrent les élites des deux régions pour la représentation légitime des Anglophones.

41

Voir Politique africaine, $\mathrm{n}^{\circ}$ 51, p. 141. 
De même, avec la libéralisation politique, le bloc musulman au Nord-Cameroun s'est également fissuré en raison de la dissociation de plus en plus faite entre ethnie peul et islam par des groupes ethniques musulmans naguère soumis à l'instar des Mandara, des Kanouri, des Haoussa, etc.

Ces rivalités trouvent généralement leur expression dans l'arène électorale ainsi que l'ont révélées les quatre consultations qui ont eu cours au Cameroun depuis la restauration du multipartisme et ce malgré les dénonciations des fraudes par l'opposition, le parti au pouvoir le RDPC (Rassemblement démocratique du peuple camerounais), étant en passe de devenir un parti ultra-dominant: les Béti et assimilés votent en majorité pour le RDPC, les Bamiléké et les Anglophones pour le SDF, les Bamoun pour l'UDC (Union démocratique du Cameroun), les Bassa pour l'UPC (Union des populations du Cameroun), toutes tendances confondues, les Peul et Kotoko pour l'UNDP, les Kirdi (exceptés les Toupouri qui votent MDR) et les Arabes choa pour le RDPC.

Les associations "culturelles" telles le Laakam bamiléké, l'Essingan béti, la DCK (Dynamique culturelle kirdi), l'ACGUI (Association culturelle guiziga), l'Association des Kanouri du Cameroun, le MOINAM (Mouvement d'investissement et d'assistance mutuelle) des Gbaya, etc., véritables groupes de pressions, en sont une autre forme d'expression. Et ce renouveau de la question ethnique nous interpelle sur le type de communauté politique à bâtir en Afrique pour notre entrée au troisième millénaire. Dans tous les cas, il importe de considérer ce continent dans tout son patrimoine matériel et spirituel, dans ses mœurs, dans ses langues et dans ses sentiments. C'est ce que Carlos Lopes ${ }^{42}$ qualifie "d'africanisation de la démocratie".

\section{De la nécessité d'un état multicommunautaire au Cameroun et en Afrique}

Comme nous venons de le voir, l'ethnicité constitue une des formes majeures de différenciation sociale et politique d'une part et d'inégalité structurelle d'autre part, des sociétés contemporaines. Elle repose sur la production et la reproduction de définitions sociales et politiques de la différence physique, psychologique et culturelle entre les groupes dits ethniques qui développent entre eux des relations des différents types(coopération, conflits, compétition, domination, reconnaissance, etc.). Donc, elle est liée à la classification sociale des individus et aux relations entre groupes dans une société donnée. Parler d'ethnicité et des groupes ethniques en isolement total est aussi absurde que de parler du bruit d'applaudissements d'une seule main. L'ethnicité ne peut émerger que 
lorsque des groupes ont un minimum de contacts entre eux et qu'ils doivent entretenir des idées de leur spécificité culturelle, physique ou psychologique réciproque afin de réduire leur existence en tant que groupe. ${ }^{43}$

Mais si l'expérience africaine et notamment camerounaise peut nous apprendre quelque chose, c'est bien que l'identité ethnique est toujours plurielle, évolutible, transformable. Elle est un construit permanent; elle est investie de sens multiples; elle est complexe parce qu'associant sans aucune exclusive des niveaux et des formes d'identité apparemment exclusifs et pourtant parfaitement vécus parce qu'en même temps cumulatifs et alternatifs. Chaque individu peut se caractériser par plusieurs identités qui se matérialisent éventuellement de façon simultanée ou successive compte tenu du contexte historique, social, économique et politique. Par exemple, il peut se sentir à la fois camerounais, sawa, francophone, anglophone, africain ou arabe dans le cas où un de ses parents est de cette origine. Il peut être porteur de ses identités simultanément ou bien choisir celle qui convient à la situation particulière dans laquelle il est plongé.

C'est cette ethnicité que depuis plus de trente ans, les partisans d'un Etat à parti unique ou de régime militaire en Afrique, se sont déployés à juguler et de promouvoir l'unité nationale pour justifier le monopole du pouvoir par une poignée de personnes. Cette position a engendré dans la plupart des cas l'avènement des dictatures et le règne de la terreur. Ces inconditionnels du monopartisme ont pensé à tort qu'il serait possible d'étouffer l'ethnicité, soit par la persuasion, soit par la contrainte, afin de modeler une nation servile. Ils se sont lamentablement trompés. ${ }^{44} \mathrm{Et}$, le renouveau de conflits ethniques, de rebellions armées et les replis identitaires qui accompagnent le processus de démocratisation est là pour l'attester. Aussi, les problèmes relatifs à la question nationale ne peuvent plus être posés de la même manière aujourd'hui qu'ils étaient pendant la colonisation. 45

En fait, le contexte ethnique constitue une dimension essentielle des modes d'organisation et de perception de soi en Afrique car, sur le plan historique, c'est le cadre ethnique ou tribal qui a assuré (et continue à assurer) la structure fondamentale de l'héritage culturel, spirituel et artistique des populations africaines. Le bagage ethnique de l'Africain moyen consiste essentiellement en un mode de vie et une perception du monde, comme peuvent

43

44

45

Cf. Martinello (n. 36), p. 18.

Oyowe, A., Un point de vue personnel: construire la démocratie sur le tribalisme, in: Le Courrier ACP-CEE, Bimestriel n 128, juillet/août 1991, p. 69.

Dieng Aly, A., Question nationale et ethnies en Afrique noire: le cas du Sénégal, papier présenté au Colloque de la $8^{\mathrm{e}}$ Assemblée générale du Codesria, Dakar, 26 juin - 2 juillet 1995, p. 1.; Martin G., La crise de l'Etat-nation en Afrique: du régionalisme au fédéralisme, in: Afrique 2000, $\mathrm{n}^{\circ} 22$, avril, mai, juin 1995 . 
l'être les structures de l'Etat dans les sociétés où la nationalité et l'élément ethnique ont fusionné en une entité commune. ${ }^{46}$ Et dans la mesure où l'ethnie a vocation à promouvoir l'intégrité politique, économique et culturelle de chacun des groupes constitutifs de la société dont elle relève, à y favoriser le retour à la confiance, à l'initiative, à la créativité sociale, à plus de solidarité et à conjurer ce faisant les forces anomiques, entropiques et centrifuges de la structure globale, elle apparaît comme un argument indispensable pour la mise en œuvre et la promotion d'un développement juste, harmonieux et par conséquent durable de l'Etat.

Donc l'actuel blocage des pays africains apparaît dans une large mesure de nature culturelle. La culture politique des populations du continent noir reste en grande partie une culture régionale, villageoise, ethnique ou religieuse, alors que les institutions en place correspondent à une culture politique tout autre, de type national. Plus concrètement le pouvoir central est privé de la base indispensable que devrait constituer une culture nationale. Ces institutions doivent alors se réconcilier avec les solidarités existantes africaines au lieu de les affronter. ${ }^{47}$ Aussi, loin d'être la cause de l'instabilité actuelle de l'Afrique, le facteur ethnique pourrait s'il est valablement géré, jouer un rôle déterminant dans l'indispensable réorganisation des Etats africains; il pourrait en effet, assurer une véritable participation aux prises de décisions politiques; car, loin d'être archaïques et rétrogrades comme certains l'ont prétendu, les racines ethniques et culturelles des peuples africains offrent une voie privilégiée de mobilisation de leur fidélité et de leur énergie en vue de l'édification des nations, à condition que la dimension ethnique de leur vie soit réellement intégrée à la structure de l'Etat.

D'ailleurs, l'on s'est délibérément servi de la culture comme instrument de l'intégration nationale, mais ce n'est pas le seul moyen d'y parvenir, ni même un moyen suffisant. Ce n'est peut-être même pas un moyen nécessaire. Par exemple, la Suisse continue d'exister alors que ses cantons sont profondément attachés aux intérêts locaux, que sa population se partage entre quatre grands groupes linguistiques, et que les " Suisses n'ont pas beaucoup investi dans la nation". En Italie, l'intégration s'est opérée sous l'effet des relations personnelles et clientélistes qui se sont instaurées entre le pouvoir central et les notables locaux, en l'absence de toute culture nationale commune. De même, les difficultés de la construction européenne sont souvent moins la conséquence d'une crispation identitaire des opinions que des divergences entre gouvernements sur le contenu et l'orientation économique de l'Union. Ces à-coups ne peuvent dissimuler le développement de l'intégration transnationale à l'échelle des régions, des entreprises ou des individus, ni les progrès réalisés dans la reconnaissance des particularismes culturels ou politiques à 
l'intérieur des Etats farouchement centralisateurs, telle l'Espagne. Nonobstant la banalisation préoccupante des thèses et de la violence racistes ou xénophobes et la montée des sentiments frileusement protectionnistes dans les domaines de l'économie ou de la culture, l'Europe ravagée par deux guerres, a su réactualiser sa tradition pluriculturelle et lui donner un fondement économique dont l'approche culturaliste est incapable de rendre compte. Et, "Aujourd'hui, affirme Jean-François Bayart, ${ }^{48}$ les perspectives d'élargissement de l'Union européenne doivent accepter ce principe de diversité".

\section{Conclusion}

Dès lors, quelles conclusions faut-il tirer de cette analyse quant au chemin à suivre par l'Afrique vers l'intégration politique?

Tout d'abord que ce n'est pas l'illusion d'un Etat-nation qui mérite plus d'attention. Le but à atteindre ici est celui de réaliser la synthèse ou mieux la réconciliation entre l'Etat postcolonial sans nation et les ethnies-nations sans Etats. C'est au prix de cette réconciliation que l'Etat et les ethnies se reconnaîtront mutuellement. Et cette reconnaissance réciproque est la condition sine qua non du dépassement de " l'Etatterritoire d'une ethnie " au profit de " l'Etat-espace politique, économique, social et culturel d'équilibre entre des individus et les ethnies" que propose Tshiyembe Mwayila ${ }^{49}$ qui suppose d'une part que l'ethnie-nation en accédant au statut de communauté, renonce à " l'espace" comme l'unique cadre de vie politique national des individus et des communautés; d'autre part, que l'Etat renonce lui aussi à toute velléité de négation ou de destruction de l'ethnie-nation. Le résultat serait l'acceptation officielle d'un Etat multicommunautaire.

C'est donc la région ou la communauté qui doit être mise au plan, c'est-à-dire en d'autres termes, la zone géographique peuplée par un groupe distinct des gens qui partagent les mêmes valeurs socio-culturelles, des moyens similaires de production et de subsistance et un attachement à un système traditionnel bien ancré d'auto-organisation. ${ }^{50}$ Cependant, cette communauté ne doit pas être purement ethnique, liant le droit du sol au droit du sang; une telle conception implique le risque d'intolérance et d'exclusion. Il faut au contraire, que dans la vie quotidienne, des populations d'origines différentes apprennent à cohabiter, à s'entraider, à coopérer, à respecter leurs différences. ${ }^{51}$ L'important est donc de trouver les

48

49

50

51

Bayart (n. 5), p. 73.

Tshiyembe Mwayila (n. 4), p. 93; Michalon (n. 4), p. 35 et seq.

Von Burgsdorff, S.K., La démocratie consociationnelle: un nouveau concept pour l'Afrique, in: Le Courrier ACP-CEE, Bimestriel, n 134, juillet/août 1992, p. 63.

Conac, G., (dir), L'Afrique en transition vers le pluralisme, Paris, Economica, 1993, p. 504 - 505. 
biais permettant pleinement de tenir compte de l'ethnicité sans lui donner institutionnellement des pouvoirs qui, eux, forcément auraient tendance à figer les groupes dont les élites voudraient s'auto-produire.

L'Etat devra alors se soucier de mettre en place un réseau scolaire de sorte que l'école serve de creuset intégrateur et qu'elle initie les agents eux-mêmes par une pédagogie adaptée aux méthodes démocratiques, lesquelles supposent un recours à la décentralisation et aux procédures de l'Etat de droit pour neutraliser les revendications ethniques, sécessionnistes ou irrédentistes. D'où le recours au modèle consociétal qui permettra aux groupes ethniques d'une part, de bénéficier d'un maximum d'autonomie en ce qui concerne leurs affaires courantes, de la proportionnalité, du consensus dans le processus décisionnel ainsi que des droits des minorités.

L'amarre a été larguée au Cameroun avec la réforme constitutionnelle du 18 janvier 1996 qui institue un Etat unitaire décentralisé en même temps qu'elle reconnaît les droits des autochtones et protège les minorités. Il reste à mettre en pratique ce train de mesures. 